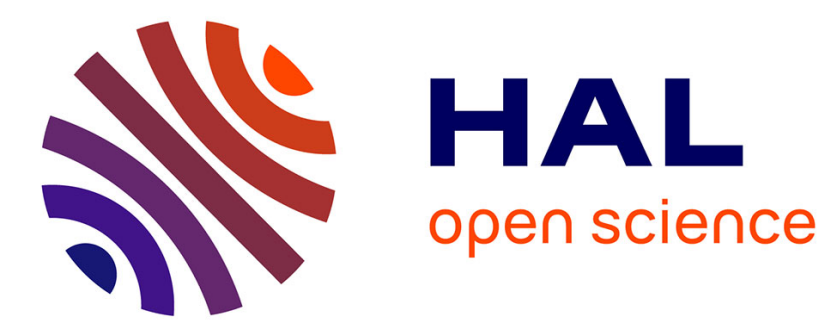

\title{
Supervisory Control of Air-Fuel Ratio in Spark Ignition Engines
}

Denis Efimov, Vladimir Nikiforov, Hossein Javaherian

\section{To cite this version:}

Denis Efimov, Vladimir Nikiforov, Hossein Javaherian. Supervisory Control of Air-Fuel Ratio in Spark Ignition Engines. Control Engineering Practice, 2014, 30 (9), pp.27-33. 10.1016/j.conengprac.2014.04.002 . hal-00974225

\section{HAL Id: hal-00974225 \\ https://hal.inria.fr/hal-00974225}

Submitted on 5 Apr 2014

HAL is a multi-disciplinary open access archive for the deposit and dissemination of scientific research documents, whether they are published or not. The documents may come from teaching and research institutions in France or abroad, or from public or private research centers.
L'archive ouverte pluridisciplinaire HAL, est destinée au dépôt et à la diffusion de documents scientifiques de niveau recherche, publiés ou non, émanant des établissements d'enseignement et de recherche français ou étrangers, des laboratoires publics ou privés. 


\title{
Supervisory Control of Air-Fuel Ratio in Spark Ignition Engines
}

\author{
Denis V. Efimov, Vladimir O. Nikiforov, Hossein Javaherian
}

Abstract' The problem of air-fuel ratio stabilization in spark ignition engines is addressed in this paper. The proposed strategy consists of proper switching among two control laws to improve quality of the closed-loop system. The first control law is based on an a priori off-line identified engine model and ensures robust and reliable stabilization of the system at large, while the second control law is adaptive, it provides on-line adaptive adjustment to the current fluctuations and improves accuracy of the closed-loop system. The supervisor realizes a switching rule between these control laws providing better performance of regulation. Results of implementation on two vehicles are reported and discussed.

\section{INTRODUCTION}

The requirement on vehicle tailpipe emissions is one of the main restrictions for engine development and certification. Three-way catalytic converters (TWC) installation in exhaust manifold aims at oxidizing HC and CO and reducing NOx concentration. Usually TWC peak efficiency is guaranteed if fuel-air ratio (FAR) is close to the stoichiometric value and the conversion efficiency of TWC is significantly reduced away from the stoichiometric value. Therefore, the primary objective of the FAR control system is to maintain the fuel injection in stoichiometric proportion to the ingested air flow (exception to this occurs in heavy load situations where a rich mixture is required to avoid premature detonation or for more power). Variations in the air flow affected by the driver serve as an exogenous disturbance to the system.

Due to its importance, the problem of FAR regulation has attracted significant attention during the last few decades [4]. Adaptive control theory [2], [7], [17], [18], robust control approaches [3], fuzzy control systems theory [8], neural network techniques [12], [19] and learning approach [1] are successfully tested in this particular application. However, the complexity of the problem and growing demands on FAR regulation quality require new solutions. These solutions have to combine reliability and performance of robust control approaches and the accuracy and insensitivity to changes of dynamics of adaptation methods. In addition, for implementation purposes, they should have a small number of tuning parameters and clear design guidelines. Switching control theory gives a solution to this problem.

There exist many good reasons and practical motivations to use a set of controllers to regulate a single plant as opposed to one controller [11], [12], [16]. In such a case the problem of trade off the advantages and disadvantages of each subsystem for modeling and control is appearing. The theory of switched systems addresses this issue proposing the proper switching laws between controllers. Application of a supervisory (switched) control algorithm may seriously improve performance of the system regulation [5]. In addition, in order to solve a complex control problem, it can be decomposed on several simpler ones with design of control laws for each of subproblems, then proper supervisor ensures switching among the controls and solution of the initial problem.

In this work, the problem of FAR regulation problem is solved considering switching between two control laws. The first one is based on robust model-based control algorithm, which ensures stability for all ranges of the system parameters and

The research was supported by the General Motors Corporation. D.V. Efimov and V.O. Nikiforov are with Department of Information and Control Systems, Saint Petersburg State Institute of Information Technology, Mechanics and Optics, Russia (nikiforov@mail.ifmo.ru). D.V. Efimov is also with Non-A project, Inria, Parc Scientifique de la Haute Borne 40, avenue Halley, 59650 Villeneuve d'Ascq, France (Denis.Efimov@inria.fr). H. Javaherian. is with Powertrain Systems Research Laboratory, GM Research and Development Center, Warren, Michigan, USA (hossein.javaherian@gm.com). 
inputs, but may have accuracy shortcomings. The second control law is adaptive, it is directed at improving the quality of transient response on a dynamic fluctuation around the reference model (used in the first control). Supervisor performs activation of the adaptive control when unsatisfactory quality of the reference model is detected and, hence, improvement of the robust control is needed. Theoretical stability conditions of the developed supervisory control are established, and the results of implementation are reported confirming efficiency of the proposed solution.

The outline of this work is as follows. In section 2 the detailed problem statement and some preliminaries are presented. Section 3 contains descriptions of the control algorithms. Supervisor equations are introduced in section 4. Results of implementation are reported in section 5. Concluding remarks are given in Section 6.

\section{Problem Statement}

It is a well-known fact that an automotive engine is a highly nonlinear multi-variable system and derivation of its precise model is a complex process. This is a reason why the simplified models of engines are very popular in practice. These models can take into account the main features of engine processes, like the presence of time delays and nonlinearities, which are important for controller design or fault detection applications. In this work nonlinear autoregressive (NARX) model is chosen for FAR dynamics description (in this context FAR refers to the non-dimensional engine-out fuel-air ratio sometimes known as $\cong$ ):

$$
y(m)=\sum_{i=1}^{k} \bar{a}_{i} y(m-i)+\sum_{j=0}^{p}\left[\overline{\mathbf{b}}_{j}^{T} \mathbf{f}(m-j)\right] u(m-j)+\sum_{j=0}^{p} \overline{\mathbf{r}}_{j}^{T} \mathbf{d}(m-j)+v(m),
$$

where $y \in R$ is FAR (the regulated output), $u \in\left[u_{\min }, u_{\max }\right]$ is the control input (fuel pulsewidth in this work, $0<u_{\min }<u_{\max }<+\infty$ are actuator constraints), $\mathbf{d} \in R^{n}$ and $\mathbf{f} \in R^{q}$ are the vectors of nonlinear input terms (may contain products of the physical engine variables, which are available for measurement like engine velocity, cam phaser positions, exhaust manifold pressure, temperatures in exhaust and intake manifolds, etc.), $k \geq 1$ and $p \geq k-1$ are the model polynomials degrees, $m$ is the number of current event (discrete time); $v \in R$ is a disturbance acting on the system; $\overline{\mathbf{a}}=\left[\bar{a}_{1} \ldots \bar{a}_{k}\right]^{T} \in R^{k}, \overline{\mathbf{B}}=\left[\overline{\mathbf{b}}_{0} \ldots \overline{\mathbf{b}}_{p}\right] \in R^{q \times(p+1)}$ and $\overline{\mathbf{R}}=\left[\overline{\mathbf{r}}_{0} \ldots \overline{\mathbf{r}}_{p}\right] \in R^{n \times(p+1)}$ are the model (1) constant parameters. The advantage of NARX model consists in availability of various methods for its approximation and simplicity of control design. It is assumed that the variables $\mathbf{d}$ and $\mathbf{f}$ are independent in the control variable $u$ and available for design, therefore, the model (1) is affine in control.

It is assumed that a dataset is given, that a priori has collected measured information on $y, u$ (and other variables involved in the vectors $\mathbf{d}, \mathbf{f}$ ) for various regimes of engine operation obtained for a preliminary control. Based on the given dataset, the compact sets $\mathcal{D} \subset R^{n}$ and $\mathcal{F} \subset R^{q}$ can be computed which define admissible values for the vectors $\mathbf{d}$ and $\mathbf{f}$ respectively. Then, applying standard approaches [15] the vectors of coefficients $\mathbf{a}, \mathbf{B}$ and $\mathbf{R}$ can be obtained as off-line approximations of $\overline{\mathbf{a}}, \overline{\mathbf{B}}$ and $\overline{\mathbf{R}}$. Substituting a, $\mathbf{B}$ and $\mathbf{R}$ in (1) we represent the dynamics of FAR loop (1) with a sufficient accuracy. The residual error can be assumed bounded and modeled as a part of the exogenous disturbance $v$. The coefficients a , B can be derived ensuring stability of the model (1) as well as stability of its inverse with respect to the control input (that corresponds to the physical nature of the engine). 
Assumption 1. Polynomials defined by the vectors of coefficients a and $\mathbf{c}$, where $c_{j}=\sum_{k=1}^{q} \mathbf{b}_{j, k}$ for $0 \leq j \leq p$, have all zeros with norms smaller than one.

Requirement on stability of the polynomial a corresponds to a physical restriction that an engine has a stable dynamics. Under this assumption and with substitution $\mathbf{f}(m-j)=1$, stabilizing controls for the system (1) can be designed applying simple inversion of its equation (inverse system is stable and, thus, the control algorithm will be realizable). The choice $\mathbf{f}(m-j)=1$ is the basic one, but some other normalized inputs may also be included in $\mathbf{f}$.

The problem is to design control $u(i) \in\left[u_{\min }, u_{\max }\right], i \geq 0$ ensuring practical output regulation to a given reference $y_{d}(i), i \geq 0$, i.e., the property $\left|y(i)-y_{d}(i)\right| \leq \Delta$ should be satisfied for all $i \geq 0$ and $\mathbf{d} \in \mathcal{D}, \mathbf{f} \in \mathcal{F}$ for some prescribed $\Delta>0$ providing that $\left|y(0)-y_{d}(0)\right| \leq \Delta$.

To this end, recall that a continuous function $\sigma: R_{+} \rightarrow R_{+}$belongs to class $\mathrm{K}$ if it is strictly increasing and $\sigma(0)=0$; additionally it belongs to class $\mathrm{K}_{\infty}$ if it is also radially unbounded; and continuous function $\beta: R_{+} \times R_{+} \rightarrow R_{+}$is from class $\mathrm{K} \mathcal{L}$, if it is from class $\mathrm{K}$ for the first argument for any fixed second one, and it is strictly decreasing to zero by the second argument for any fixed first one.

\section{CONTROL AlgORIthmS}

In this section description of robust model-based and adaptive controls are presented.

\section{A. Model-based control algorithm}

The following is the condition of the control applicability.

Assumption 2. For all $\mathbf{f} \in \mathcal{F}$ it holds $\mathbf{b}_{0}^{T} \mathbf{f} \neq 0$.

Since the vector $\mathbf{f}$ is composed by measured engine variables or their nonlinear functions and products, which all have some sets of admissible values, then assumption 2 can be easily checked for $\mathbf{f} \in \mathcal{F}$ and the vector of coefficients $\mathbf{b}_{0}$. For instance, $\mathbf{f}(i), i \geq 0$ and elements of $\mathbf{b}_{0}$ can be all positive (that may be guaranteed by proper approximation of (1)).

Under assumptions 1 and 2, the control law is calculated as a simple inversion of the model (1) with respect to $u$ :

$$
U(m)=\frac{1}{\mathbf{b}_{0}^{T} \mathbf{f}(m)}\left[y_{d}(m)-U_{P I D}(m-1)-\sum_{i=1}^{k} a_{i} y(m-i)-\sum_{j=0}^{p} \mathbf{r}_{j}^{T} \mathbf{d}(m-j)-\sum_{j=1}^{p}\left[\mathbf{b}_{j}^{T} \mathbf{f}(m-j)\right] u(m-j)\right],
$$

where due to the presence of the disturbance $v$ (which reflects possible unmodeled dynamics, measurement noise and approximated model errors) it is required to use an internal feedback in the form of a nonlinear PID:

$$
U_{P I D}(m)=k_{1} e(m)+k_{2} \sum_{i=0}^{m} e(i)+k_{3}[e(m)-e(m-1)]+k_{4} \operatorname{sign}(e(m))+k_{5} e(m)^{3},
$$

where $e=y_{d}-y$ is the regulation error, $k_{j}, j=\overline{1,5}$ are control parameters, which have to be determined based on real or computer experiments. The terms proportional to $k_{1}$ and $k_{5}$ are responsible for proportional feedback ( $k_{1}$ for local regulation, and $k_{5}$ for big deviations of $e(m)$, appearance of two gains helps to improve quality of feedback). The terms proportional to $k_{2}$ and $k_{3}$ correspond to integral and differential actions respectively. The term with $k_{4}$ allows small matched 
disturbances to be compensated. The control (2) under substitution $u(m)=U(m)$ ensures the model inversion and the following closed loop dynamics:

$$
y(m)=y_{d}(m)-U_{P I D}(m-1)+v(m) .
$$

Without $U_{P I D}$ the control (2) forms the so-called feedforward part of the regulator, that does not contain any feedback errors (it depends on the current and past values of the inputs and outputs of the engine dynamics and the approximated coefficients of the model).

The control (2) cannot be realized in practice since there exist constraints on admissible control amplitudes, i.e. it should be within the following bounds: $u_{\min } \leq u \leq u_{\max }$. The implementation of a simple saturation

$$
u(m)=\operatorname{sat}[U(m)], \operatorname{sat}(x)=\left\{\begin{array}{l}
u_{\min } \text { if } x<u_{\min } ; \\
u_{\max } \text { if } x>u_{\max } \\
x \text { otherwise, }
\end{array}\right.
$$

for stable plants provides a solution to the problem. Define the control actuator error as follows

$$
\delta(m)=\mathbf{b}_{0}^{T} \mathbf{f}(m)[u(m)-U(m)],
$$

then the closed loop dynamics of (1), (4) takes the form:

$$
\begin{aligned}
& y(m)=y_{d}(m)-U_{P I D}(m-1)+\tilde{v}(m), \\
& \tilde{v}(m)=v(m)+\delta(m) .
\end{aligned}
$$

Prop os ition 1 . Under assumptions 1 and 2 there exist constants $k_{j}, j=\overline{1,5}$ such that for any solutions of the system (1), (2), (3), (4) with $\mathbf{d} \in \mathcal{D}, \mathbf{f} \in \mathcal{F}$ for all $i \geq 0$ :

$$
|e(i)| \leq \beta_{1}(|e(0)|, i)+\gamma_{1}\left(\|\tilde{v}\|_{[0, i]}\right),\|\tilde{v}\|_{[0, i]}=\sup _{0 \leq j \leq i}|\tilde{v}(j)|, \beta_{1} \in \mathrm{K} \mathcal{L}, \gamma_{1} \in \mathrm{K} .
$$

Proof. From assumption 1 the system (1) produces bounded solutions for any bounded control $u_{\min } \leq u \leq u_{\max }$ and $\mathbf{d} \in \mathcal{D}, \mathbf{f} \in \mathcal{F}$. Moreover, the equations (5) can be rewritten as follows

$$
e(m)=k_{1} e(m-1)+k_{2} \sum_{i=0}^{m-1} e(i)+k_{3}[e(m-1)-e(m-2)]+k_{4} \operatorname{sign}(e(m-1))+k_{5} e(m-1)^{3}-\tilde{v}(m) .
$$

Particularly, for $\left|k_{1}\right|<1$ and $k_{2}=k_{3}=k_{4}=k_{5}=0$ this system becomes linear and it has the estimate

$$
|e(i)| \leq|e(0)| \exp \left[\ln \left(k_{1}\right) i\right]+\left(1-k_{1}\right)^{-1}\|\tilde{v}\|_{[0, i]}, i \geq 0,
$$

which is an example of the desired one.

Additionally, adjusting values of the coefficients $k_{j}, j=\overline{2,5}$ one can improve the performance of the control (4). For example, coefficient $k_{2}$ provides insensitivity to static errors (integral part of PID), coefficient $k_{4}$ cancels disturbances with amplitudes less than $k_{4}$, and coefficient $k_{5}$ may help on large deviations of the error.

Proposition 1 establishes a qualitative result on the error convergence, the estimate obtained in proposition 1 is close to input-to-state stability property introduced in the paper [9]. 


\section{B. Adaptive control algorithm}

The control (4) has feedbacks aimed at attenuation of disturbances, approximation errors and measurement noise. In spite of this, in some cases an additional adaptation of the control is further needed. An important issue is that the model (1) has been approximated on a large a priori collected dataset, and the coefficients $\mathbf{a}, \mathbf{B}$ and $\mathbf{R}$ suit well for all $\mathbf{d} \in \mathcal{D}, \mathbf{f} \in \mathcal{F}$. However for some operating conditions, which were not well presented in the dataset, there exists another set of coefficients $\tilde{\mathbf{a}}, \tilde{\mathbf{B}}$ and $\tilde{\mathbf{R}}$ which represents dynamics of FAR more accurately. In fact, for almost all modes of engine operation there exist such coefficients locally working better than the global ones $\mathbf{a}, \mathbf{B}$ and $\mathbf{R}$.

Thus the problem of the coefficients $\tilde{\mathbf{a}}, \tilde{\mathbf{B}}$ and $\tilde{\mathbf{R}}$ identification with posterior update of the control can be posed. To solve the problem it is proposed to use the conventional identification algorithm [6] denoting

$$
y(m)=\sum_{i=1}^{k} \tilde{a}_{i} y(m-i)+\sum_{j=0}^{p}\left[\tilde{\mathbf{b}}_{j}^{T} \mathbf{f}(m-j)\right] u(m-j)+\sum_{j=0}^{p} \tilde{\mathbf{r}}_{j}^{T} \mathbf{d}(m-j)=\omega(m) \theta,
$$

where $\theta=\left[\begin{array}{lllll}\tilde{\mathbf{a}}^{T} & \tilde{\mathbf{b}}_{0}^{T} & \ldots \tilde{\mathbf{b}}_{p}^{T} & \tilde{\mathbf{r}}_{0}^{T} & \ldots \\ \tilde{\mathbf{r}}_{p}^{T}\end{array}\right]^{T}$ and

$$
\omega(m)=\left[y(m-1) \ldots y(m-k) \mathbf{f}(m)^{T} u(m) \ldots \mathbf{f}(m-p)^{T} u(m-p) \mathbf{d}(m)^{T} \ldots \mathbf{d}(m-p)^{T}\right]
$$

is the row of regressors. Then we obtain the following parameterization for the identification error

$$
\varepsilon(m)=y(m)-\hat{y}(m)=\omega(m)[\theta-\hat{\theta}(m)],
$$

where $\hat{\theta}=\left[\begin{array}{lllll}\widehat{\mathbf{a}}^{T} \hat{\mathbf{b}}_{0}^{T} \ldots \hat{\mathbf{b}}_{p}^{T} & \hat{\mathbf{r}}_{0}^{T} \ldots \hat{\mathbf{r}}_{p}^{T}\end{array}\right]^{T}$ is the adjustable vector of estimates for $\theta$ and $\hat{y}(m)$ is the output of the adaptive observer:

$$
\widehat{y}(m)=\sum_{i=1}^{k} \widehat{a}_{i}(m) y(m-i)+\sum_{j=0}^{p} \widehat{\mathbf{r}}_{j}^{T}(m) \mathbf{d}(m-j)+\sum_{j=0}^{p}\left[\widehat{\mathbf{b}}_{j}^{T}(m) \mathbf{f}(m-j)\right] u(m-j) .
$$

Note that the model (6) has a form similar to (1). However, for the coefficients $\tilde{\mathbf{a}}, \tilde{\mathbf{B}}$ and $\tilde{\mathbf{R}}$ it is assumed that $v(i)=0$, $i \geq 0$ (the coefficients locally approximate the system dynamics exactly). The observer (7) also has the form (1) under $\theta$ substitution instead of $\hat{\theta}$, thus $\hat{y}(m)$ is an estimate of the output $y(m)$.

To design the adaptation algorithm for $\hat{\theta}$ let us choose the conventional quadratic error functional $Q_{\varepsilon}(m)=0.5 \varepsilon(m)^{2}$, whose minimization is ensured by the following gradient adaptation algorithm [6]:

$$
\hat{\theta}(m)=\hat{\theta}(m-1)+\gamma(m) \omega(m-1)^{T} \varepsilon(m-1),
$$

where $\gamma(m)>0$ is a design parameter. To specify conditions of the algorithm (8) applicability we impose the following restrictions on the engine dynamics.

Assumption 3 . For all $\mathbf{d} \in \mathcal{D}, \mathbf{f} \in \mathcal{F}$ it holds:

Б there exist series $\tilde{\mathbf{a}}_{k}, \tilde{\mathbf{B}}_{k}$ and $\tilde{\mathbf{R}}_{k}$ and $\Delta_{k} \geq 0, k \geq 0$ such that the model (6) is valid with $\tilde{\mathbf{a}}_{k}, \tilde{\mathbf{B}}_{k}$ and $\tilde{\mathbf{R}}_{k}$ for all $j_{k} \leq i \leq j_{k+1}$ for all $k \geq 0$ with $j_{k+1}=j_{k}+\Delta_{k}, j_{0}=0$;

5 for any $i \geq 0$ there exist $0 \leq K\left(K<\Delta_{k}\right.$ for all $\left.k \geq 0\right), \varsigma>0$ and $0<\rho<1$ such that

$$
\prod_{j=i}^{i+K-1} P_{j} \leq \rho \mathbf{I}, P_{j}=\mathbf{I}-\left(\varsigma+|\omega(j)|^{2}\right)^{-1} \omega(j)^{T} \omega(j),
$$


where $\mathbf{I}$ is the identity matrix of the corresponding dimension.

This assumption has two parts. First, it is assumed that the time range of the system operation can be decomposed on subintervals $j_{k} \leq i \leq j_{k+1}, k \geq 0$, where the model (6) is valid for some $\tilde{\mathbf{a}}_{k}, \tilde{\mathbf{B}}_{k}$ and $\tilde{\mathbf{R}}_{k}$. Secondly, a variant of persistency of excitation condition is introduced, that is required for the convergence of the adjusted parameters to $\theta_{k}=\left[\tilde{\mathbf{a}}_{k}^{T} \tilde{\mathbf{b}}_{0, k}^{T} \ldots \tilde{\mathbf{b}}_{p, k}^{T} \tilde{\mathbf{r}}_{0, k}^{T} \ldots \tilde{\mathbf{r}}_{p, k}^{T}\right]^{T}$. This part can be verified numerically on-line (during experiments/implementation) or offline on the given a priori dataset.

Proposition 2. In the observer (7) with the adaptation algorithm (8) for $\gamma(m)=\left(\varsigma+|\omega(j)|^{2}\right)^{-1}$, the parameter identification error $\tilde{\theta}_{k}(i)=\hat{\theta}(i)-\theta_{k}$ has the following properties:

$\mathrm{B}$ if the first part of Assumption 3 holds, then $\left|\tilde{\theta}_{k}(i+1)\right| \leq\left|\tilde{\theta}_{k}(i)\right|, j_{k} \leq i \leq j_{k+1}, k \geq 0$;

$\mathrm{B}$ if Assumption 3 holds, then the estimate is satisfied:

$\left|\tilde{\theta}_{k}(i)\right| \leq\left|\tilde{\theta}_{k}\left(j_{k}\right)\right| \exp \left[\ln (\rho)\left(i-j_{k} \bmod K\right)\right], j_{k} \leq i \leq j_{k+1}, k \geq 0$.

Proof. From (8) we have

$$
\hat{\theta}(m)-\theta_{k}=\hat{\theta}(m-1)-\theta_{k}+\gamma(m) \omega(m-1)^{T} \varepsilon(m-1),
$$

and the identification error has form $\varepsilon(m-1)=y(m-1)-\hat{y}(m-1)=\omega(m-1)\left[\theta_{k}-\hat{\theta}(m-1)\right]$. Then

$$
\tilde{\theta}_{k}(m)=\tilde{\theta}_{k}(m-1)-\left(\varsigma+|\omega(j)|^{2}\right)^{-1} \omega(m-1)^{T} \omega(m-1) \tilde{\theta}_{k}(m-1)=P_{m-1} \tilde{\theta}_{k}(m-1) .
$$

The matrices $P_{m-1}=\mathbf{I}-\left(\varsigma+|\omega(j)|^{2}\right)^{-1} \omega(m-1)^{T} \omega(m-1)$ have one singular value between 0 and 1 (the corresponding eigenvector is $\omega(m-1)$, and other singular values are equal to one (the case $P_{m-1} \tilde{\theta}_{k}(m-1)=\tilde{\theta}_{k}(m-1)$ is equivalent to $\varepsilon(m-1)=0)$. This fact means that the error $\tilde{\theta}_{k}$ is not an increasing function of time. From (9) we have:

$$
\tilde{\theta}_{k}(m+K)=\prod_{j=1}^{K} P_{m+j-1} \tilde{\theta}_{k}(m-1)
$$

and $\left|\tilde{\theta}_{k}(m+K)\right| \leq \rho\left|\tilde{\theta}_{k}(m-1)\right|$ from assumption 3, which implies the estimate.

Under conditions of proposition 2, the parametric error $\tilde{\theta}_{k}(m)$ asymptotically converges to zero, then taking control

$$
\begin{aligned}
& u(m)=\operatorname{sat}\left(U_{a}(m)\right), U_{a}(m)=\frac{1}{\widehat{\mathbf{b}}_{0}^{T} \mathbf{f}(m)}\left[y_{d}(m)-U_{P I D}(m-1)-\sum_{i=1}^{k} \widehat{a}_{i} y(m-i)-\right. \\
& \left.-\sum_{j=0}^{p} \widehat{\mathbf{r}}_{j}^{T} \mathbf{d}(m-j)-\sum_{j=1}^{p}\left[\widehat{\mathbf{b}}_{j}^{T} \mathbf{f}(m-j)\right] u(m-j)\right]
\end{aligned}
$$

it is possible to ensure the model (6) stabilization, where $U_{P I D}(m-1)$ is defined by (3).

Proposition 3 . Under assumption 3 there exist constants $k_{j}, j=\overline{1,5}$ such that for any solution of the system (1), (7), (8), (10) with $\mathbf{d} \in \mathcal{D}, \mathbf{f} \in \mathcal{F}$ for all $j_{k} \leq i \leq j_{k+1}, k \geq 0\left(\beta_{2} \in \mathrm{K} \mathcal{L}, \gamma_{2} \in \mathrm{K}\right)$ :

$|e(i)| \leq \beta_{2}\left(\left|e\left(j_{k}\right)\right|, i-j_{k}\right)+\gamma_{2}\left(\left|\tilde{\theta}_{k}\left(j_{k}\right)\right|\right)$.

Pro of. Since the control $u(m)=W(m)+w(m)$, where 


$$
w(m)=u(m)-W(m), W(m)=\frac{1}{\tilde{\mathbf{b}}_{0}^{T} \mathbf{f}(m)}\left[\begin{array}{c}
y_{d}(m)-U_{P I D}(m-1)-\sum_{i=1}^{k} \tilde{a}_{i} y(m-i)- \\
-\sum_{j=0}^{p} \tilde{\mathbf{r}}_{j}^{T} \mathbf{d}(m-j)-\sum_{j=1}^{p}\left[\tilde{\mathbf{b}}_{j}^{T} \mathbf{f}(m-j)\right] u(m-j)
\end{array}\right],
$$

the system (1), (10) has the following closed loop dynamics:

$$
e(m)=k_{1} e(m-1)+k_{2} \sum_{i=0}^{m-1} e(i)+k_{3}[e(m-1)-e(m-2)]+k_{4} \operatorname{sign}(e(m-1))+k_{5} e(m-1)^{3}-\tilde{w}(m)
$$

with $\tilde{w}(m)=\mathbf{b}_{0}^{T} \mathbf{f}(m) w(m)$. Then as in proposition 1 there exist functions $\beta_{2} \in \mathrm{K} \mathcal{L}$ and $\gamma \in \mathrm{K}$ such that

$$
|e(i)| \leq \beta_{2}\left(\left|e\left(j_{k}\right)\right|, i-j_{k}\right)+\gamma\left(\|\tilde{w}\|_{\left[j_{k}, i\right]}\right)
$$

for all $j_{k} \leq i \leq j_{k+1}, k \geq 0$. Since for all $\mathbf{d} \in \mathcal{D}, \mathbf{f} \in \mathcal{F}$ there exists $g>0$ such that $|\tilde{w}(m)| \leq g\left|\tilde{\theta}_{k}(m)\right|$, then $\|\tilde{w}\|_{\left[j_{k}, i\right]}=g\left|\tilde{\theta}_{k}\left(j_{k}\right)\right|$, that implies the result for $\gamma_{2}(s)=\gamma(g s), s \geq 0$.

The advantage of the control (10) is that $\tilde{\theta}_{k}(i) \rightarrow 0$ with $i \rightarrow \infty$ according to proposition 2, therefore, if the adaptive algorithm is active for sufficiently long time (constants $\Delta_{k} \geq 0$ from assumption 3 are large enough), then the adaptive control (7), (8) and (10) ensures an exact regulation of FAR dynamics at the stoichiometric value.

In this section two control algorithms are proposed, which ensure input-to-state stabilization of FAR dynamics. Both controls are based on the measurement information (the first one designed for the approximated off-line model, the second one for the on-line model). The issue of the improvement of the closed-loop system quality with the use of special switching between these control laws is discussed in the following section.

\section{THE SUPERVISOR}

All proposed control algorithms from section 3 possess their own advantages. The control algorithm designed off-line is rather reliable (it ensures stability for all operating modes of the engine) and robust (it is not sensitive to disturbances and unmodeled dynamics), but it may fail to ensure good accuracy over the entire range of operating conditions. The adaptive control has some transients after which it is tuned to compensate all errors at a particular engine operating condition. The switching algorithm executed in the supervisor has to combine the advantages of these controls neglecting their shortages providing the closed loop control with an improved performance. For this purpose note that the main difference between these controls consists in the models on which they are based. The following performance functionals evaluate accuracy of the models on the last $L \geq 0$ steps:

$$
\begin{aligned}
& J_{1}(m)=L^{-1} \sum_{q=m-L}^{m} e_{1}^{2}(q), J_{2}(m)=L^{-1} \sum_{q=m-L}^{m} e_{2}^{2}(q), \\
& e_{1}(q)=y(q)-\sum_{i=1}^{k} a_{i} y(q-i)-\sum_{j=0}^{p}\left[\mathbf{b}_{j}^{T} \mathbf{f}(q-j)\right] u(q-j)-\sum_{j=0}^{p} \mathbf{r}_{j}^{T} \mathbf{d}(q-j), \\
& e_{2}(q)=y(q)-\sum_{i=1}^{k} \widehat{a}_{i}(m) y(q-i)-\sum_{j=0}^{p}\left[\widehat{\mathbf{b}}_{j}^{T}(m) \mathbf{f}(q-j)\right] u(q-j)-\sum_{j=0}^{p} \widehat{\mathbf{r}}_{j}^{T}(m) \mathbf{d}(q-j) .
\end{aligned}
$$




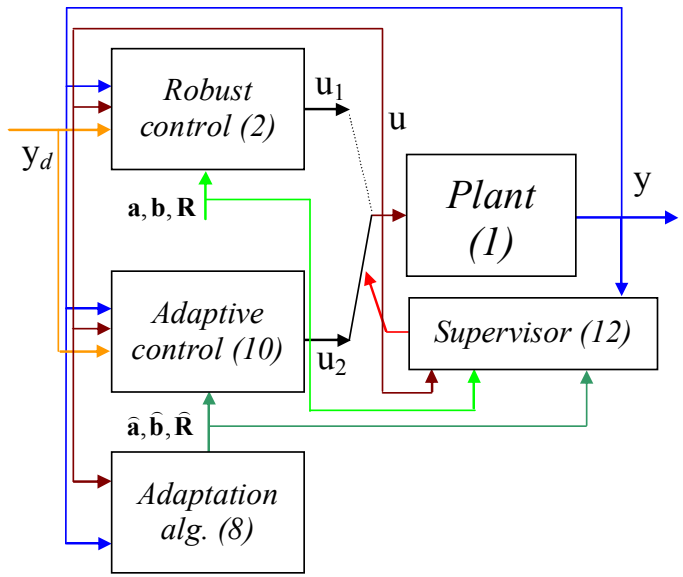

Fig. 1. The structure scheme of the supervisory control system of the engine

In this case activation of the control with the most accurate model looks reasonable, that is the idea of supervision algorithm in this work. The width of the time window $L$ used for evaluation of accuracy of both models is a design parameter (it is also related with the rate of convergence of the adaptive observer (7), (8)). However, the switching among nonlinear systems is not so trivial. Even if the systems are asymptotically stable or input-to-state stable as in our case, an inappropriate switching strategy may lead to instability [13].

The problem of switching among input-to-state systems has been addressed in the previous works [5], [10], [11]. The main idea there consists in the dwell-time mechanism application. Dwell-time constant restricts the rate of switching between the controls and for a sufficiently slow rate the stability of the closed loop system is guaranteed. For the rest of the section let $u_{1}(m)$ be defined by (2) and $u_{2}(m)$ be given as in (10). The structure scheme of the control system is presented in Fig. 1.

Th e or e $\mathrm{m} 1$. Let assumptions 153 hold and for some $0<\lambda<1$

Б there exist dwell-time constant $\tau_{D}>0$ such that $\beta_{i}\left(r, \tau_{D}\right) \leq \lambda r$ for all $r \geq 0$;

Б $\tau_{D}<\Delta \leq \Delta_{k}$ for all $k \geq 0$ for some $\Delta>0$;

$\bar{B} \bar{\beta}(r) e^{\ln (\lambda) \Delta / \tau_{D}} \leq \lambda^{2} r$ for all $k \geq 0$ and $r \geq 0$, where $\bar{\beta}(s)=\max \left\{\beta_{1}(s, 0), \beta_{2}(s, 0)\right\}$.

If $s_{w+1}-s_{w} \geq \tau_{D}, w \geq 0$, where $s_{w}$ is the instant of $w^{\text {th }}$ switch, then in the system (1) with the control

$u(m)=u_{z\left(s_{w}\right)}(m), z\left(s_{w}\right) \in\{1,2\}$

for any solutions the following estimate is satisfied for some $\beta \in \mathrm{K} \mathcal{L}$ and $\gamma \in \mathrm{K}$ :

$$
|e(i)| \leq \beta(|e(0)|, i)+\gamma\left(\|l\|_{[0, i]}\right), l(i)= \begin{cases}\mid \tilde{v}_{(i)} \text { if } & i \geq s_{w}, z\left(s_{w}\right)=1 ; \\ \left|\tilde{\theta}_{k}\left(s_{w}\right)\right| & \text { if } i \geq s_{w}, z\left(s_{w}\right)=2 .\end{cases}
$$

Proof. Consider $j_{k} \leq i \leq j_{k+1}$. Since assumptions 1 and 2 hold, while $z\left(s_{w}\right)=1, w \geq 0$ the estimate

$$
|e(i)| \leq \beta_{1}\left(\left|e\left(s_{w}\right)\right|, i-s_{w}\right)+\gamma_{1}\left(\|\tilde{v}\|_{\left[s_{w}, i\right]}\right),\|\tilde{v}\|_{\left[s_{w}, i\right]}=\sup _{s_{w} \leq j \leq i}|\tilde{v}(j)|, \beta_{1} \in \mathrm{K} \mathcal{L}, \gamma_{1} \in \mathrm{K}
$$

from proposition 1 is true. Under assumptions 1 and 3 the estimate from proposition 3 is valid for all $z\left(s_{w}\right)=2, w \geq 0$ :

$$
|e(i)| \leq \beta_{2}\left(\left|e\left(s_{w}\right)\right|, i-s_{w}\right)+\gamma_{2}\left(\left|\tilde{\theta}_{k}\left(s_{w}\right)\right|\right),
$$


where $\tilde{\theta}_{k}(m)=\hat{\theta}(m)-\theta_{k}$ with $j_{k} \leq s_{w} \leq j_{k+1}$ for all $k \geq 0, \beta_{2} \in \mathrm{K} \mathcal{L}, \gamma_{2} \in \mathrm{K}$. According to Lemma 2 of [5] for dwelltime switched input-to-state systems, the upper estimate follows for all $j_{k} \leq i \leq j_{k+1}$ and all $k \geq 0$ :

$$
|e(i)| \leq \lambda^{-1} \bar{\beta}\left(\left|e\left(j_{k}\right)\right|\right) e^{\ln (\lambda)\left(i-j_{k}\right) / \tau_{D}}+(1-\lambda)^{-2} \bar{\gamma}\left(\|l\|_{[0, i]}\right), \bar{\gamma}(s)=\max \left\{\gamma_{1}(s), \gamma_{2}(s)\right\} .
$$

Now we may use the same technique considering $\Delta_{k} \geq \Delta>0$ as a 'dwell-time_ between switches among different operating modes of the system, that under the theorem conditions gives:

$$
|e(i)| \leq \lambda^{-2} \bar{\beta}(|e(0)|) e^{\ln (\lambda) i / \Delta}+(1-\lambda)^{-4} \bar{\gamma}\left(\|l\|_{[0, i]}\right),
$$

that implies the desired estimate.

The stated restriction $\tau_{D}<\Delta$ implies that switching between the controls in the supervisor must be faster than the change in the operating regime. The dwell-time switching algorithm ensures boundedness of the system trajectories and the theorem presents the worst-case estimate on the closed loop error behavior. Dwell-time switching algorithm still leaves room to further supervisor rule design focusing on the improvement of transient behavior. Keeping in mind the performance functionals (11) and dwell-time rule from theorem 1, the following supervision algorithm is proposed:

$$
\begin{aligned}
& u(m)=u_{z\left(s_{w}\right)}(m), z\left(s_{w}\right) \in\{1,2\}, w \geq 0, \\
& s_{w+1}= \begin{cases}\arg \inf _{m \geq s_{w}+\tau_{D}}\left\{J_{2}(m)<J_{1}(m)\right\} & \text { if } s_{w}=1 ; \\
\arg \inf _{m \geq s_{w}+\tau_{D}}\left\{J_{1}(m)<J_{2}(m)\right\} & \text { if } s_{w}=2,\end{cases} \\
& z\left(s_{w+1}\right)=3-z\left(s_{w}\right), z\left(s_{0}\right)=1, s_{0}=0,
\end{aligned}
$$

where $s_{w}, w \geq 0$ determines the time instant of the last switch; $\tau_{D}>0$ is the dwell-time that prevents chattering (high frequency switching between the control algorithms) and ensures the closed-loop stability. For $s_{0}=1$ the system starts with the model-based control (2), if accuracy of the adaptation model (7) is better $\left(J_{2}(m)<J_{1}(m)\right)$, then the adaptive control (10) has to be activated. If after dwell-time the accuracy of the model (1) again becomes better $\left(J_{1}(m)<J_{2}(m)\right)$ the control (2) would be switched on giving time for adaptation algorithm to adjust the observer.

An additional logic can be added to (12) for an emergent switching back to control $u_{1}$, when the regulation error with the control $u_{2}$ in the loop becomes suddenly high. In this case a kind of average dwell-time condition would be ensured, under which the result of theorem 1 can be confirmed after a mild modification of arguments. Such a modification is omitted for brevity of presentation.

\section{Application Results}

The proposed switching control has been tested in two vehicles with V8 engines: Chevrolet Tahoe with 5.7£ engine and GMC Yukon with a 5.3^ engine (these vehicles and such a type of engines are rather popular on US market, in addition, the emission improvement for vehicles with a big volume of engines is of great practical importance). The schedule of testing was as follows. 

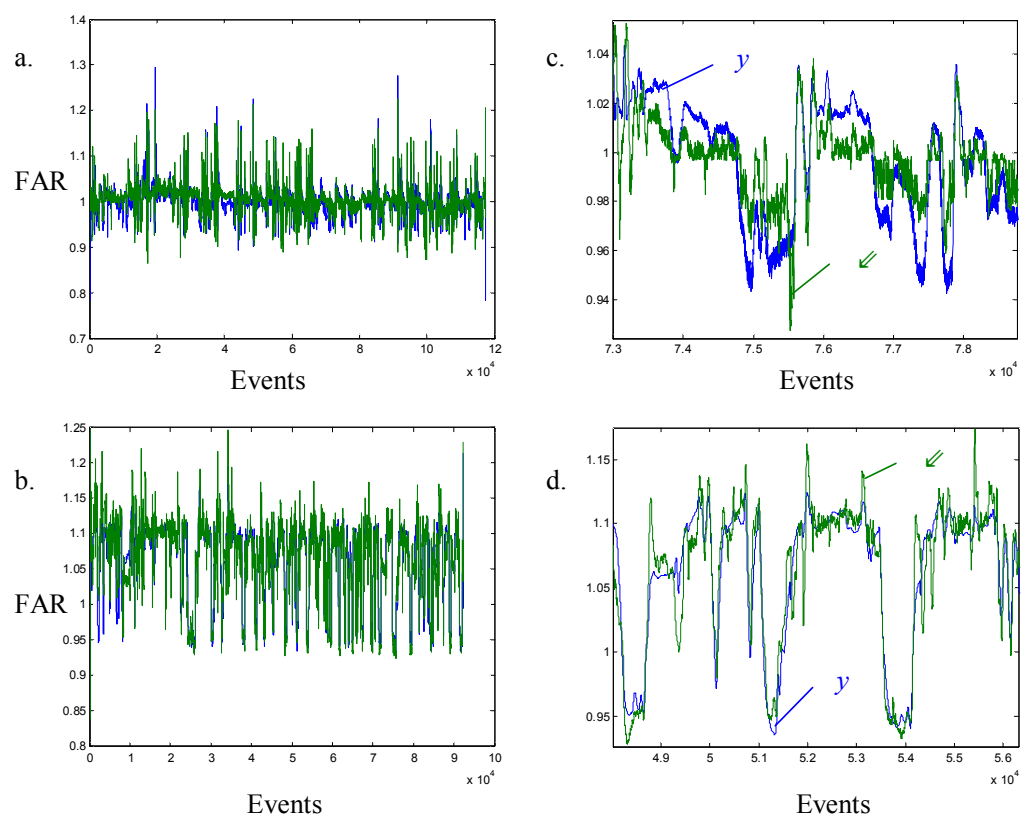

Fig. 2. FAR model accuracy verification

At the first step based on the databases of previous experiments, the model (1) was derived for both vehicles (the coefficients $\mathbf{a}, \mathbf{B}$ and $\mathbf{R}$ satisfy assumption 1). The results of model (1) tests are shown in Fig. 2.a, 2.c and Fig. 2.b, 2.d for $5.7 \uparrow$ and $5.3 \uparrow$ engines, respectively (the figures $2 . c$ and $2 . d$ demonstrate zoomed plots of the figures 2.a and 2.b). Fulfillment of the persistency of excitation condition (the second part of assumption 3) has been verified on both datasets. As we can deduce from these plots the quality of both models are of sufficient accuracy for the control (2) design.

At the second step the controls (2) and (10) are calculated. Assumption 2 is verified for the control (2) on the a priori collected dataset. Coefficients $k_{j}, j=\overline{1,5}$ are chosen zero initially, and after some experimentations they are tuned to some values providing acceptable performance. For the control (10) the same values of coefficients $k_{j}, j=\overline{1,5}$ are chosen. The assumption 3 is valid (after a verification on the dataset). After that, the system is ready for experimental testing.

During the experiments, the control algorithms and the supporting schemes have been loaded in the board computer using dSpace, where these codes have replaced the production controllers. Next, tests in different operating conditions (e.g. Federal Test Program) have been performed. The sampling time is time-varying and it is proportional to the speed of engine rotation.

The results of the tests are shown in figures 3 and 4 for the vehicles with the $5.7 \uparrow$ and $5.3 \uparrow$ engines, respectively (the parts (a) and (b) represent the variables $e_{F A R}=F A R-1$ and $u$ respectively (in these experiments 1 was selected as the desired value for the fuel-air ratio, which corresponds to the stoichiometric value), the part (c) plots the values of the functionals $J_{1}$ and $J_{2}$ ). In these results, the supervisor switches between two control algorithms based on quality functionals, the instants of switches almost everywhere correspond to verification of the conditions (12) (dwell-time constant has been assigned sufficiently small). The adaptive regulator provides better quality of control of FAR dynamics. A change in modes of engine operation results in backward activation of the model (1) based controls (in Fig. 3 the adjusted model has almost 
everywhere a better accuracy, in Fig. 4 in some cases activation of the control $u_{1}$ happens, while the adaptive observer needs a time for adjustment after an operating mode change). For both vehicles, experiments confirm applicability of the proposed approach. A comparison of the obtained results with a reference controller implemented in both vehicles shows a good potential of the proposed supervisory controller.
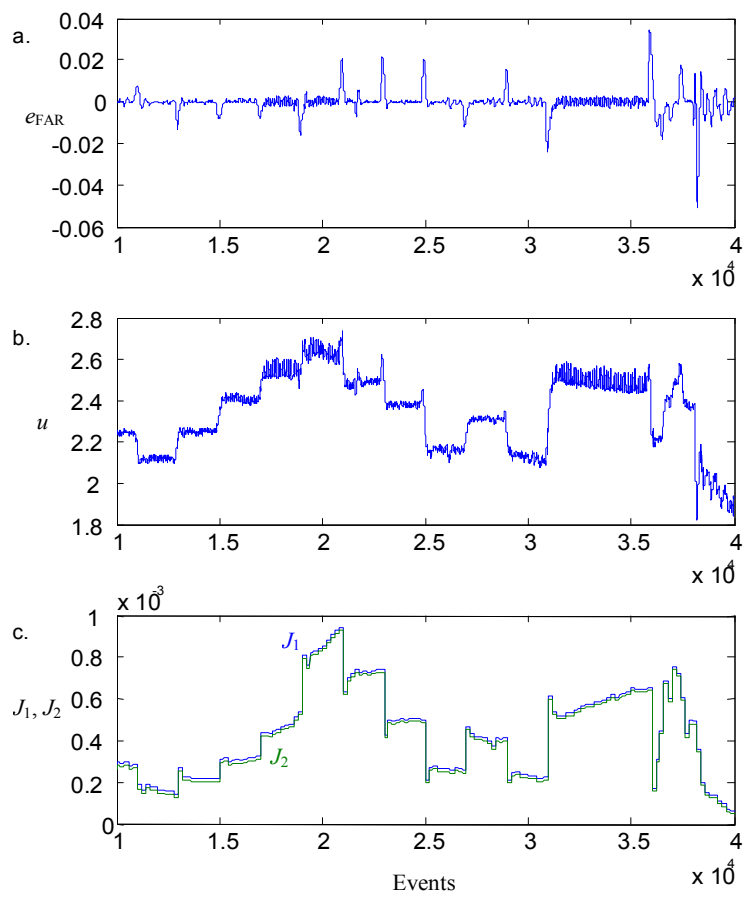

Fig. 3. Trajectories for the vehicle with a $5.7 \uparrow$ engine

\section{CONCLUSION}

Switching control algorithm for air-fuel ratio regulation is developed and practically tested for two vehicles. The controller contains three parts: robust model-based control, adaptive control and the supervisor. The first control is designed for approximated off-line model using a priori available experimental dataset. The adaptive control is based on an adjusted in real-time model. Both models and controls have similar structure and the only difference is the type of information used for their design (off-line or on-line measurements). The supervisor provides switching between these controls taking into account the current accuracy of the models. If off-line approximated model has better quality, then the robust control is active. If the adaptively adjusted model has better accuracy of FAR dynamics representation during some number of previous events, then the adaptive control is switched on. Such a scheme allows us to combine the reliability of robust control (which was intensively tested and it ensures admissible quality of FAR regulation for all operating regimes) and the flexibility of the adaptive control (which can improve the performance due to the higher local accuracy of the FAR dynamics approximation). Stability conditions are established. Practical implementation and intensive tests demonstrate applicability of the approach. Augmentation of an anti-windup scheme for the developed control can be a subject of future researches. 

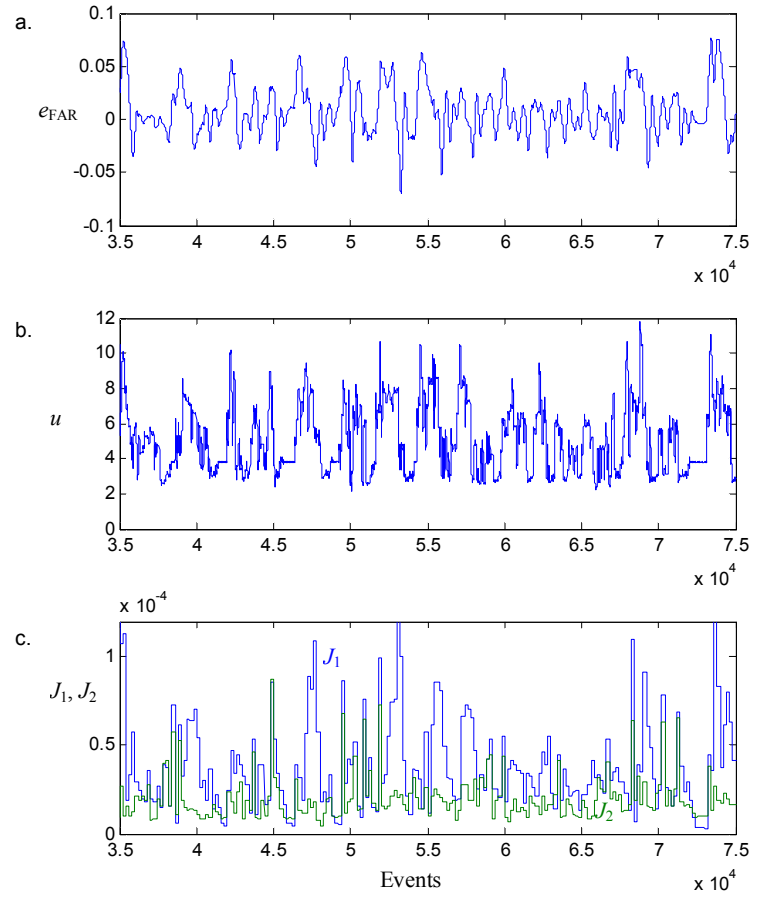

Fig. 4. Trajectories for the vehicle with a $5.3 \uparrow$ engine

\section{ACKNOWLEDGMENT}

We would like to thank Dr. Man-Feng Chang of GM R\&D for his continued support of the project and many useful discussions during the course of this study.

\section{REFERENCES}

[1] Andrianov D.I., Manzie C., Brear M.J. (2013). Spark ignition engine control strategies for minimising cold start fuel consumption under cumulative tailpipe emissions constraints. Control Engineering Practice, 21(8), pp. 1007Б1019.

[2] Ault B.A., Jones V.K., Powell J.D., Franklin G.F. (1993). Adaptive air-fuel ratio control of a spark ignition engine. SAE paper No. 940373.

[3] Brandstetter M. (1996). Robust Air-Fuel Ratio Control For Combustion Engines. Cambridge, UK, Ph.D. thesis.

[4] Cook J.A., Kolmanovsky I.V., McNamara D., Nelson E.C., Prasad K.V. (2007). Control, computing and communications: Technologies for the Twenty-First century Model T. Proc. of the IEEE, 95, pp. 3345355.

[5] Efimov D.V., Panteley L., Loria A. (2008). On Input-to-Output Stability of Switched Nonlinear Systems. Proc. 17th IFAC WC, Seoul, Korea, pp. 3647Б3652.

[6] Fradkov A.L., Miroshnik I.V., Nikiforov V.O. (1999). Nonlinear and Adaptive Control of Complex Systems. Dordrecht, Boston, London: Kluwer Academic Publishers.

[7] Franceschi E.M., Muske K.R., Peyton-Jones J.C., Makki I.H. (2007). An Adaptive Delay-Compensated PID Air Fuel Ratio Controller. Proc. SAE-2007-01-1342, World Congress \& Exhibition, Detroit.

[8] Ghaffari A., Shamekhi A.H., Saki A., Kamrani E. (2008). Adaptive Fuzzy Control for Air-Fuel Ratio of Automobile Spark Ignition Engine. Proc. World Acad. Science, Engineering, Technology, 12, pp. 1064Б1072. 
[9] Jiang Z-P., E.D. Sontag and Wang Y. (1999). Input-to-state stability for discrete-time nonlinear systems. Proc. 14th IFAC World Congress (Beijing), Vol. E, pp. 277-282.

[10] Hespanha J.P., Liberzon D., Morse A.S. (2002). Supervision of integral-input-to-state stabilizing controllers. Automatica, 38(8), pp. 132761335.

[11] Hespanha J.P., Morse A.S. (1999). Certainty equivalence implies detectability. Syst. Controls Lett., 36, pp. 1 Б13.

[12] Huang T., Liu D., Javaherian H. and Sin N. (2008). Neural sliding mode control of engine torque, Proc. 2008 IFAC Triennial World Congress, Seoul, South Korea.

[13] Liberzon D. (2003). Switching in Systems and Control. Systems and Control: Foundations and Applications. Boston, MA: Birkhauser.

[14] Liu D., Javaherian H., Kovalenko O. and Huang T. (2008). Adaptive critic learning techniques for engine torque and air-fuel ratio control, IEEE Transactions on Systems, Man and Cybernetics, Part B: Cybernetics, 38(4), pp. 988Б993.

[15] Ljung L. (1999). System Identification: Theory for the User (second ed.). Prentice-Hall, Englewood Cliffs, NJ.

[16] Morse A.S. (1995). Control using logic-based switching. In: Trends in control (A. Isidori (Ed.)), Springer-Verlag, pp. $69^{-} 113$.

[17] Powell J.D., Fekete N.P., Chang C.-F., (1998). Observer-based air fuel ratio control. IEEE Control Systems Magazine, 18(5), pp. 72583.

[18] Turin R.C., Geering H.P. (1995). Model-reference adaptive A/F-ratio control in an SI engine based on Kalman-filtering techniques. Proc. American Control Conference, pp. 408254090.

[19] Zhai Y., Yu D. (2007). RBF based feedforward feedback control for air-fuel ratio of SI engines. Proc. IFAC Workshop on Advanced Fuzzy and Neural Control. 